Europhys. Lett., 59 (3), pp. 344-350 (2002)

\title{
Critical properties of excitation waves on curved surfaces: Curvature-dependent loss of excitability
}

\author{
V. A. Davydov ${ }^{1}\left(^{*}\right)$, N. $\operatorname{Manz}^{2,3}$, O. $\operatorname{Steinbock}^{3}$ and S. C. MÜller $\left.{ }^{2}{ }^{* *}\right)$ \\ 1 Department of Physics, MIREA - Vernadskogo 78, 117454 Moscow, Russia \\ 2 Institut für Experimentelle Physik, Otto-von-Guericke-Universität \\ D-39106 Magdeburg, Germany \\ 3 Department of Chemistry and Biochemistry, Florida State University \\ Tallahassee, FL 32306-4390, USA
}

(received 11 February 2002; accepted in final form 6 May 2002)

PACS. 05.45.-a - Nonlinear dynamics and nonlinear dynamical systems.

PACS. 82.20.Wt - Computational modeling; simulation.

\begin{abstract}
In the literature, different properties of propagating excitation waves on curved surfaces are published. Theoretical papers predicted critical properties of waves on curved surfaces. If an excitation wave propagates in a non-planar system, its geodetic curvature causes a transition from excitable to non-excitable dynamics. In this paper we present first experimental results of the transition in a weakly excitable BZ system which are in good agreement with the theoretical predictions.
\end{abstract}

Introduction. - Excitation wave patterns occur in many physical, chemical, and biological systems [1-3]. Up to now, most investigations were done in planar geometries. But in nature there are many two-dimensional systems which must be considered as an excitable medium with a curved surface. An important example is the heart, where the excitation waves move along the muscle in a ring-like fashion. Although during the last decade some properties of excitation fronts on curved surfaces were studied theoretically [4-6] and experimentally [7-9], there are still many gaps in our understanding of processes in non-planar systems. An important aspect, especially in biological systems, is the study of possible mechanisms for controlling these waves. Recently, new qualitative properties of excitation fronts on curved surfaces have been predicted and confirmed by computer simulations [6]. In particular, it has been shown that under certain conditions a curved surface can express critical properties, i.e., excitation fronts with sufficiently large geodetic curvature will be broken in a weakly excitable medium. Moreover, it was shown that some surfaces (for example, "bottle-like" surfaces) can act as a diode being "transparent" for wave propagation only in one direction, but not in the other. Note that the diode effect can also be observed in inhomogeneous planar excitable media [10] and in photosensitive BZ systems [11]. In this paper we describe the first experimental observations of critical properties of curved surfaces, such as breaking of wave fronts, in using a periodically curved system with a weakly excitable BZ reaction.

$\left({ }^{*}\right)$ E-mail: davydov@lpi.ac.ru

$\left({ }^{* *}\right)$ E-mail: stefan.mueller@physik.uni-magdeburg.de

(c) EDP Sciences 
Excitable media on curved surfaces. - Wave patterns in an excitable medium are usually described by non-linear reaction-diffusion equations. In this work we consider a commonly used two-variable model of excitable two-dimensional media. For waves propagating on a curved surface, the basic equations can be written in the form

$$
\begin{aligned}
& \frac{\partial u}{\partial t}=F_{1}(u, v)+D_{u} \frac{1}{\sqrt{g}} \frac{\partial}{\partial \alpha^{i}}\left(g^{i k} \sqrt{g} \frac{\partial u}{\partial \alpha^{k}}\right), \\
& \frac{\partial v}{\partial t}=\epsilon F_{2}(u, v)+D_{v} \frac{1}{\sqrt{g}} \frac{\partial}{\partial \alpha^{i}}\left(g^{i k} \sqrt{g} \frac{\partial v}{\partial \alpha^{k}}\right)
\end{aligned}
$$

where $u\left(\alpha^{1}, \alpha^{2}, t\right)$ and $v\left(\alpha^{1}, \alpha^{2}, t\right)$ are referred to as the activator and inhibitor species, respectively, $\alpha^{i}$ are the coordinates on the surface, and $g=\operatorname{det}\left[g_{i k}\right]$ is the determinant of the metric tensor $g_{i k}$. From here on the summation over repeated indices is implied. The functions $F_{1}$ and $F_{2}$ specify reactions in the system, and the formal rate coefficient $\epsilon$ determines the time scale for the recovery process (in many cases of interest $\epsilon$ can be regarded as a small parameter). The second terms on the right-hand sides of eqs. (1) represent the diffusive contributions, where $D_{u}$ and $D_{v}$ are the diffusion coefficients of both species. Equations (1) are complicated and, in general, they can be solved only numerically, when the functions $F_{1}$ and $F_{2}$ are specified.

For an analytical study one can use the "kinematic approach" [5]. In the framework of the kinematic theory a chemical wave is completely determined by specifying the line of its front. The form and evolution of the excitation front can be described by its natural equation $k_{\mathrm{g}}=k_{\mathrm{g}}(l, t)$, where $l$ is the arclength, $t$ is the time, $k_{\mathrm{g}}$ is the geodetic curvature. The geodetic curvature of a line on a curved surface corresponds to the curvature of its projection onto a tangential surface. Each part of the front moves in normal direction with the velocity $V=V\left(k_{\mathrm{g}}\right)$. The free end of a broken front can grow or contract with tangential velocity $C$, where $k_{\mathrm{g} 0}$ is the geodetic curvature of the front at the free end. The main kinematic equation for the evolution of the front on a curved surface has the following form [4]:

$$
\frac{\partial k_{\mathrm{g}}}{\partial l}\left(\int_{0}^{l} k_{\mathrm{g}} V \mathrm{~d} \xi+C\right)+\frac{\partial k_{\mathrm{g}}}{\partial t}+\frac{\partial^{2} V}{\partial l^{2}}+k_{\mathrm{g}}^{2} V=-\Gamma V .
$$

$\Gamma$ denotes the Gaussian curvature of the surface, which can be calculated by the multiplication of the two main curvatures in a point of the surface.

In ref. [6] it could be shown, by computer simulations, that for sufficiently small $k_{\mathrm{g}}$ and if the width of the activator front $b$ satisfies the inequality $\left|\frac{\mathrm{d} k_{\mathrm{g}}}{\mathrm{d} l}\right| b \ll\left|k_{\mathrm{g}}\right|$, the dependence of $V$ on $k_{\mathrm{g}}$ is linear, analogous to the "normal" eikonal equation in planar systems:

$$
V=V_{0}-D_{u} k_{\mathrm{g}}
$$

$V_{0}$ is the velocity of the wave front with zero geodetic curvature. This equation has been suggested previously [4] on the basis of intuitive arguments and used to develop the kinematic theory of excitation waves on curved surfaces. For large geodetic curvatures the dependence of $V$ on $k_{\mathrm{g}}$ becomes non-linear.

We now recall that for excitation waves on a plane there exists a critical curvature $K^{*}$ above which a stable propagation becomes impossible [12]. The same effect occurs on curved 


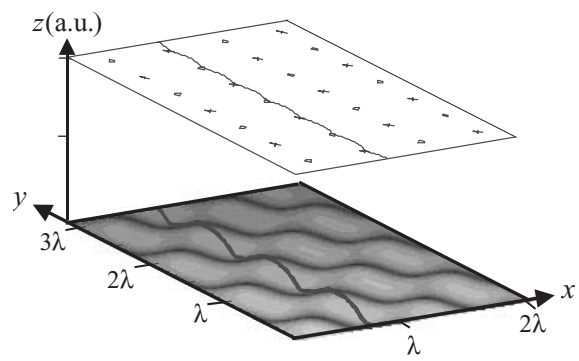

Fig. 1 - Schematic drawing of an excitation front (lower thick line) propagating in the periodically curved BZ system (eq. (5)) with small modulation of the surface and the projection of the front on a plane at $z=$ const. Because of the small modulation, the projection corresponds to the geodetic curvature of the front in eq. (7). The diamonds and crosses in the plane indicate local maxima and minima of the surface, respectively.

surfaces. In this case stable wave propagation requires $k_{\mathrm{g}}<K^{*}$, i.e. the geodetic curvature $V\left(k_{\mathrm{g}}\right)$ of the front should not exceed the critical curvature $K^{*}[6]$ and one gets, as a necessary condition for wave damping,

$$
k_{\mathrm{g}}>K^{*}
$$

Thus, the critical curvature $K^{*}$ is an important parameter for an excitable medium determining the stability of wave propagation and conditions of their damping. When propagating on a curved surface, the wave front can reach a region, where its geodetic curvature will exceed the critical curvature $K^{*}$. In this case the front will be broken. The expression for the critical curvature can be written as

$$
K^{*}=\eta \frac{V_{0}}{D_{u}}, \quad \eta<1,
$$

where the coefficient $\eta$ is an increasing function of the excitability. One can see from eq. (4) that there are two ways to obtain damping - to decrease the excitability or (and) to decrease the velocity $V_{0}$.

Critical properties of a periodically curved surface. - Let us consider the evolution of an initially flat front on a medium with slightly and periodically modulated surface characterized by amplitude $A$ and wave vector $b=2 \pi / \lambda$ :

$$
z=A \sin (b x) \sin (b y), \quad A b \ll 1,
$$

where $x, y, z$, are the Cartesian coordinates. The Gaussian curvature of the surface (5) can be written as $[9]$

$$
\Gamma(x, y)=-\frac{A^{2} b^{4}}{2}(\cos (2 b x)+\cos (2 b y)) .
$$




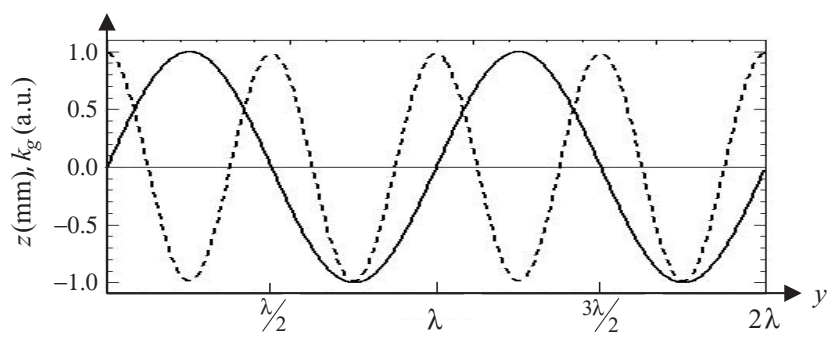

Fig. 2 - Relation between the modulated surface along the $y$-axis calculated by eq. (5) at $x=\lambda / 4(-)$ and the geodetic curvature of a front passing through this surface in the $x$-direction at this position, which is normalized to $1(---)$ calculated by eq. (7). The damping regions with the highest geodetic curvature can be found over the nodes $z=0$ of the surface at $n \lambda / 2$.

It is the same in the minima and the maxima and will induce periodic deformations of an initially flat front. These deformations were analytically studied by making use of the main kinematic equation (2) and the eikonal equation (3) and compared with experimental results [9]. The expression for the geodetic curvature of the front propagating in an arbitrary direction on the curved surface was in a very good agreement with the experimental results. A schematic drawing of the front shape and its projection on a plane is shown in fig. 1.

For the case corresponding to a wave propagating along the $x$-axis, the expression for the geodetic curvature is the following:

$$
k_{\mathrm{g}}=\frac{A^{2} b^{2} V_{0}}{8 D_{u}}\left(\cos (2 b y)+\frac{2 b D_{u}}{V_{0}} \cos \left(2 b V_{0} t\right)\right) .
$$

For the surface used in the experiments, $2 b D_{u} / V_{0} \ll 1$ and we can neglect the second term which describes small temporal oscillations.

If the amplitude of the spatial oscillations of geodetic curvature (7) exceeds the critical curvature $K^{*}$, one can expect the damping of the front near the $y$-locations:

$$
y=\frac{n \pi}{b}=\frac{n \lambda}{2},
$$

where $n$ is an integer number. The condition for damping can be written as

$$
\eta<\frac{A^{2} b^{2}}{8}
$$

Experimental setup. - The idea to observe the damping of wave fronts on a periodically curved surface was as follows: It is well known that in a closed system the excitability of the $\mathrm{BZ}$ reaction decreases in time. With decreasing parameter $\eta$ we can expect that after some time its value will be so reduced that the condition (9) will be satisfied and we should be able to observe the damping. In the experiments described in [9] we used fresh reaction solution and thus no critical phenomena as considered in this work were observed.

For the experiments in this paper and in [9] we used an acrylic glass mold $(100 \times 100) \mathrm{mm}^{2}$ with two complementary surfaces, that create a thin hollow space with a constant thickness $\xi$ in normal direction of $(61.5 \times 61.5 \times 0.4) \mathrm{mm}^{3}$ to accommodate the chemical-reaction solution. 


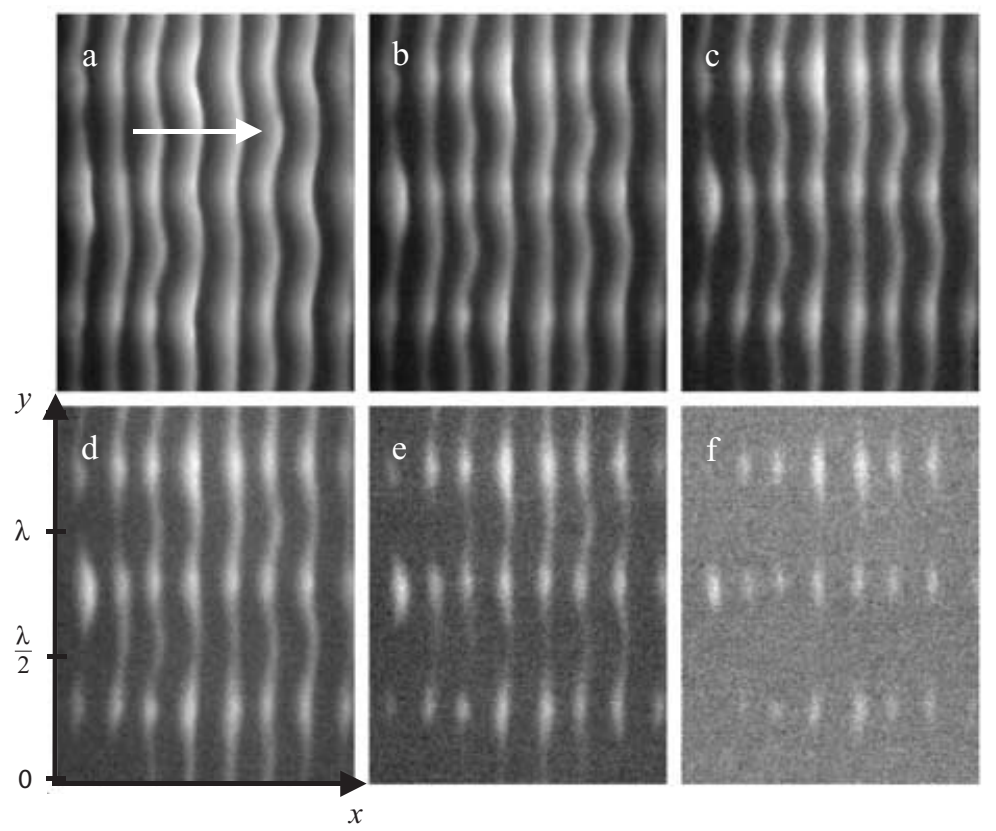

Fig. 3 - The transition from excitability to non-excitability depends on the geodetic curvature $k_{\mathrm{g}}$ of the front. In the neighborhood of the nodes of the surface $(z=0)$ at $y=n \lambda / 2$ the transition ceases earlier. Around the extrema at $y=(2 n+1) \lambda / 4$, with negative geodetic curvature, the waves are disappearing later. The arrow in (a) indicates the propagation direction of the waves. Time intervals between the consecutive images: $\Delta t=10.0 \mathrm{~s}$. Field of view: $(10.7 \times 13.9) \mathrm{mm}^{2}$.

At one side the acrylic glass mold has a channel to fill the system. Centered in the hollow, quadratic area the periodically curved region with $(31.5 \times 31.5) \mathrm{mm}^{2}$ was placed. One surface of the curved region is defined by eq. (5),

$$
z_{1}(x, y)=A \sin (b x) \sin (b y)
$$

with an amplitude $A=1.00 \mathrm{~mm}$ as well as a wavelength $\lambda=9.00 \mathrm{~mm}$ and a wave vector $b=0.70 \mathrm{~mm}^{-1}$ of the surface undulations. The surface of the second side at a constant distance in normal direction of $\xi=0.40 \mathrm{~mm}$ is calculated as

$$
z_{2}(\tilde{x}, \tilde{y})=A \sin (b \tilde{x}) \sin (b \tilde{y})+\xi /(A b)^{2},
$$

with $\tilde{x}=x-[\xi \cos (b x) \sin (b y)] / \chi$ and $\tilde{y}=y-[\xi \sin (b x) \cos (b y)] / \chi$. In this case the normalization is $\chi=\left[1 / 2-(1 / 2) \cos (2 b x) \cos (2 b y)+1 /(A b)^{2}\right]^{1 / 2}$.

Because of the small distance between the two surfaces, $z_{1}$ and $z_{2}$, we now have a quasitwo-dimensional system that can be described by the explicit function (5). If one plots eq. (5) over the extrema in the $y$-direction $\left(e . g ., x=\frac{\lambda}{4}\right)$ and the geodetic curvature of a front at this position one gets, according to fig. 2, the expected damping regions as predicted in eq. (8). Over the nodes of the surface $(z=0)$ at $y=n \lambda / 2$ the geodetic curvature calculated by eq. (7) is maximal.

The classical organic substrate (malonic acid) of the ferroin-catalyzed BelousovZhabotinsky solution was replaced by 1,4-cyclohexanedione (1,4-CHD) to prevent the forma- 

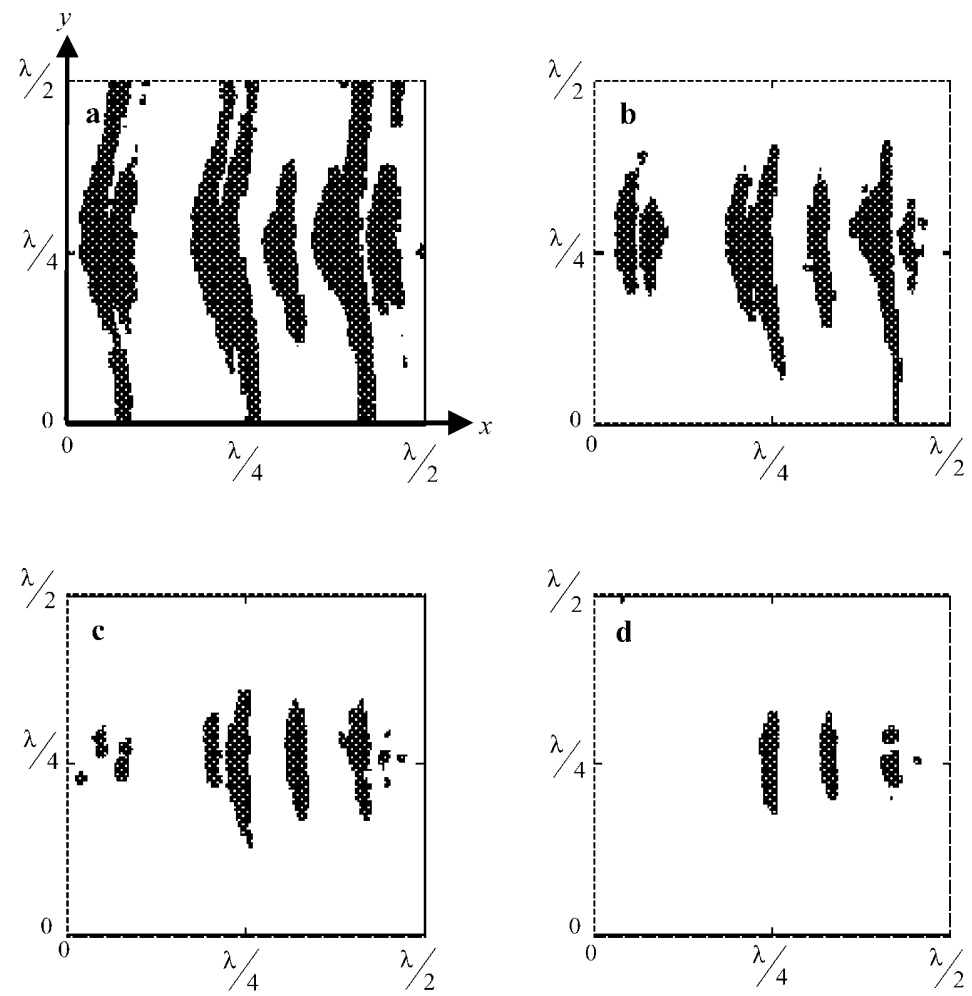

Fig. 4 - Superposition of 8 different waves propagating from left to right around a local extremum of the surface at $(\lambda / 4, \lambda / 4)$. The 4 images with $\triangle t=5.0 \mathrm{~s}$ show the delayed transition over the extrema with the lowest value of the geodetic curvature of a front.

tion of undesired $\mathrm{CO}_{2}$ bubbles in this closed system $[13,14]$. The stock solutions of $\mathrm{NaBrO}_{3}$ (Fluka) and 1,4-CHD (Sigma-Aldrich) were prepared in bidistilled water. $\mathrm{H}_{2} \mathrm{SO}_{4}$ (5 M, Fisher) and ferroin $(25 \mathrm{mM}$, Fluka) were used without further treatment. The solution with the initial concentrations of $\left[\mathrm{H}_{2} \mathrm{SO}_{4}\right]_{0}=1.50 \mathrm{M},\left[\mathrm{NaBrO}_{3}\right]_{0}=0.08 \mathrm{M},[1,4-\mathrm{CHD}]_{0}=0.12 \mathrm{M}$ and [ferroin $]_{0}=0.6 \mathrm{M}$ was filled approximately $50 \mathrm{~min}$ after preparation into the acrylic glass mold. The wave initiation was generated about $30 \mathrm{~min}$ later at the location of a straight silver wire placed outside the curved part in the planar region between the two inner surfaces of the acrylic glass mold. The differences in the absorption spectra of the reduced solution state and the oxidized wave were monitored with a monochrome charge-coupled-device (CCD) camera (COHU 2122) to detect the wave propagation [15]. The video signal was digitized using an image-acquisition card (Data-Translation DT3155, 640 pixel $\times 480$ pixel, 8 bit) and sampled with a rate of 0.5 frames/s with the commercial software HLImage ++97 . The dynamics of the wave patterns was analyzed on a personal computer with programs written in IDL (Interactive Data Language, from Research System Inc., version 5.1.1 and 5.3.1).

Experimental results. - The damping of wave fronts can be seen in fig. 3 at $y=n \lambda / 2$ as predicted in eq. (8). The originally continuous wave fronts with a wavelength of $\lambda_{\mathrm{w}}=2,8 \mathrm{~mm}$ are divided above the nodes $(z=0)$ of the surface. The fronts can propagate, if the geodetic curvature of the front is $k_{\mathrm{g}}<K^{*}$. With increasing time, more and more parts of the fronts switch into the non-excitable state and the single front segments are shrinking until the whole 
front is damped out.

Figure 4 shows a superposition of eight waves at different minima and maxima, each plotted in the $x$ and $y$ directions from 0 to $\lambda$. In image 4a some waves are still propagating over the nodes at $y=0$ and $y=\lambda$. With increasing time and decreasing excitability the waves are damped from the nodes, where the front has the highest geodetic curvature, to the extremum with the lowest geodetic curvature of the front. So the condition for damping in eq. (9) is valid for more and more segments of the wave fronts until the whole waves are vanished.

Conclusions. - Theoretical investigations of excitation waves on curved surfaces describe many properties which are not yet experimentally verified. For theoretical analysis we used a kinematic equation linearized for the case of a slightly deformed surface. New details can be obtained after solving the main kinematical equation (2) or after carrying out numerical simulations with the two-variable model of eqs. (1). The critical property that the wave propagation depends on the geodetic curvature of the front was predicted in [6]. In this paper we presented first experimental results to prove the predicted behaviour. Wave propagation in a weakly excitable, non-planar BZ system will induce deformations of an initially flat wave front. With increasing time, the excitability and, because of that, the critical curvature are decreasing. Then the different geodetic curvatures along the modulated wave front can exceed the critical curvature at defined locations. At these front parts, which are growing in time, the wave is damped until the whole front is vanished.

$$
* * *
$$

This work was supported by the Deutsche Forschungsgemeinschaft, the Florida State University and the US CRDF-RF Ministry of Education, Award VZ-010-0. We thank H. ŠEvČÍkOVÁ for fruitful discussions.

\section{REFERENCES}

[1] Field R. J. and Burger M. (Editors), Oscillations and Traveling Waves in Chemical Systems (John Wiley \& Sons, New York) 1985.

[2] Holden A. V., Markus M. and Othmer H. G. (Editors), Nonlinear Wave Processes in Excitable Media (Plenum, New York) 1991.

[3] Epstein I. R. and Pojman J. A. (Editors), An Introduction to Nonlinear Chemical Dynamics (Oxford University Press, Oxford, New York) 1998.

[4] Brazhnik P. K, Davydov V. A. and Mikhailov A. S., Theor. Math. Phys., 74 (1988) 300.

[5] Davydov V. A., Zykov V. S. and Mikhailov A. S., Sov. Phys. Usp., 34 (1991) 665.

[6] Davydov V. A., Morozov V. G. and Davydov N. V., Phys. Lett. A, 267 (2000) 326.

[7] Maselko J. and Showalter K., Nature, 339 (1989) 609.

[8] Steinbock O., Phys. Rev. Lett., 78 (1997) 745.

[9] Davydov V. A., Manz N., Steinbock O., Zykov V. S. and Müller S. C., Phys. Rev. Lett., 85 (2000) 868.

[10] Agladze K. I., Aliev R. R., Yamaguchi T. and Yoshikawa K., J. Phys. Chem., 100 (1996) 13895.

[11] Tóth A., Horváth D. and Yoshikawa K., Chem. Phys. Lett., 345 (2001) 471.

[12] Zykov V. S., Biophysics, 25 (1980) 906.

[13] Kurin-Csörgei K., Szalai I., Molnár-Perl I. and Körös E., React. Kinet. Catal. Lett., 53 (1994) 115.

[14] Kurin-Csörgei K., Szalai I. and Körös E., React. Kinet. Catal. Lett., 54 (1995) 217.

[15] Müller S. C., Plesser Th. and Hess B., Physica D, 24 (1987) 71. 